\title{
Evaluating the Location of Tsunami Sensors: Methodology and Application to the Northeast Coast of Taiwan
}

\author{
Guan-Yu Chen ${ }^{*}$ and Chin-Chu Liu \\ Institute of Applied Marine Physics and Undersea Technology, National Sun Yat-Sen University, Kaohsiung 80424, Taiwan, ROC
}

Received 31 March 2008, accepted 4 August 2008

\begin{abstract}
The "max gain" which is the maximum absolute value of the reciprocal Green's Function of a city vulnerable to tsunami damage is used to evaluate the efficiency of the location for tsunami detecting instruments. The max gain is the correlation between the water elevation variations at the observatory and the vulnerable coastal city. After vulnerable cities are determined, the max gain diagram can be used to determine the most favorable location for observation.

To verify the applicability of the methodology of a tsunami monitoring system, the Marine Cable Hosted Observatory (MACHO) project in Taiwan is analyzed in detail. Four vulnerable cities, Tou-cheng, Chi-long, Su-ao, and Hua-lien, are chosen first. It is found that from the hydrodynamic point of view the Ryukyu Island Arc is more important than the Yaeyama Ridge. The max gain distribution is found to be useful for optimizing the tsunami monitoring system in that some modification on the node locations is predicted to significantly improve the efficiency of the tsunami sensors without changing the route or the cost.
\end{abstract}

Key words: Reciprocal Green's function, Tsunami sensor, Max gain

Citation: Chen, G. Y. and C. C. Liu, 2009: Evaluating the location of tsunami sensors: Methodology and application to the northeast coast of Taiwan. Terr. Atmos. Ocean. Sci., 20, 563-571, doi: 10.3319/TAO.2008.08.04.01(T)

\section{INTRODUCTION}

The south Asian tsunami of December, 2004 has highlighted the vulnerabilities of coastal regions to tsunamis and serves as a reminder of the importance of preventive measures. To protect coastal residents from a devastating tsunami, numerous observation projects have been devoted to providing early warning for tsunamis along the Pacific Rim.

Since most tsunamis are generated by submarine earthquakes, a marine observatory is very important. Seismological information can help people to prepare for an upcoming tsunami as early as possible; the in situ water surface elevation is essential for the determination of tsunami height and excludes the possibility of a false alarm.

Because the cost of deploying and maintaining seismometers and tsunami sensors is very high, their locations should be carefully chosen so that the instruments can be used effectively. A tsunami's wave height in the deep ocean is small, and hence not easy to measure. If a hazardous tsunami has a very small amplitude at some offshore point $\mathrm{B}$, it

\footnotetext{
* Corresponding author

E-mail: guanyu@faculty.nsysu.edu.tw
}

will be hard to detect and hence point B is not a favorable location for tsunami monitoring. Clearly, the optimal position for monitoring a tsunami will be where the tsunami wave height is most closely related to the tsunami height at the hazard-prone city, which is, however, a hydrodynamic consideration not properly included in most observation projects.

In this paper, a methodology is proposed based on numerical simulation to evaluate the efficiency of seismometers and tsunami sensors. After the location of the most vulnerable cities are determined, reciprocal Green's functions are computed to determine the max gain, which is the maximum elevation change of the hazard-prone coasts following a tsunami generated with unit height at the sea point of the sensor.

\subsection{Green's Function (GF) and Its Application in Tsunami Studies}

Traditionally, Green's function (GF) is the response of a linear system to a concentrated forcing, usually a Dirac delta 
function, at a specific source. In the case of tsunami, the GF is the fundamental solution of the linear shallow water Eq. (1) which will be further explained in section 2.2. Within general boundary conditions, there is no analytic GF formulation for Eq. (1) and the GF has to be solved numerically. The Dirac delta function cannot be used because numerical calculations cannot treat infinity. Thus, the numerical evolution of unit amplitude at the point source is used as the GF.

Once the GF is computed for every point in the source zone, the response of any point of interest in the computational domain can be obtained simply by superposing the product of all source elevations and the corresponding GF. Thus, with the aid of previously computed GF's, a tsunami can be forecast very quickly because no equation-solving is involved. Note that although a tsunami wave is highly nonlinear nearshore, it can be adequately described by linear shallow water equations when the ocean is deeper than $50 \mathrm{~m}$ (Shuto 1991). In combination with a linear least square inversion, GF can also be used to retrieve the fault deformation in the source zone from the tsunami record of tidal gauges (Satake 1987).

The above-mentioned GF is the response of the whole domain to the forcing at one source point, or of a onesource-all-receiver type ( $\mathrm{Xu}$ 2007). This traditional GF is from $s$ to $r$, where $s$ is the location of the source point, and $r$ is the location of the hazard-prone city (receiver). Its reciprocal GF means the response at $s$ for the unit elevated water surface (forcing) at the hazard-prone city $r$.

Loomis (1979) noted that the traditional GF and the reciprocal GF are symmetric with respect to $s$ and $r$. That is, the response at $r$ due to a unit forcing at $s$ is exactly the response at $s$ due to a unit forcing at $r$. This reciprocity property was proposed by Loomis as an efficient method for calculating GF: by computing the reciprocal GF based on the unit elevation at a specific point of interest $r$ and recording the time series of every grid point in the ocean, from any source region the GF at $r$ is obtained. Consequently, the tsunami height at $r$ can be obtained simply by multiplication and superposition.

$\mathrm{Xu}$ (2007) proposed an all-source GF, which is exactly the reciprocal GF, to hasten the calculation process in tsunami forecasting. The "max gain," which means the maximum absolute value of the surface elevation at each sea point, is proposed to characterize the response. In the present study, the max gain is used to evaluate the efficiency of the location of tsunami sensors and seismometers.

\subsection{Marine Cable Hosted Observatory (MACHO) Project}

Located in the northeast of Taiwan, the Marine Cable Hosted Observatory (MACHO) is a recently proposed project designed to provide early warning for earthquakes and tsunamis. The cable route for the MACHO project starts from the coastal city Tou-cheng, at the western end of the Ryukyu Island Arc. As is shown in Fig. 1, the cable extends southeastward along the Ryukyu Island Arc and two junction boxes (nodes) are designed for connecting instruments. Then, the cable turns southward to the Yaeyama Ridge and two more nodes are situated along the ridge. Afterwards, the cable route shifts back to the Ryukyu Island Arc and ends at Tou-cheng, completing a loop. The preliminary route and proposed locations of the four nodes are shown in Fig. 1. The longitude and latitude of the four nodes are shown in Table 1.

The budget for the MACHO project is more than ten million US dollars, much more than the total fund devoted to tsunami research by the National Science Council, the government agency that supports most scientific activities of Taiwan, in the last thirty years. To make the best use of this project, it is worthwhile to study the efficiency of each of the node locations. Based on the results of a numerical tsunami model, a methodology for evaluating instrument locations to find the optimum location is proposed in the present study. This approach applies the max gain of the reciprocal GF to the MACHO project, which is analyzed as an example.

\section{METHODOLOGY}

A tsunami observatory is implemented to protect as many people as possible from hazardous tsunamis. For example, a coastal city with a dense population should be considered as a point of importance; the more people who are protected, the more effective the observation system will be.

However, two cities of similar population may suffer different levels of destruction because the local bathymetry is different. To determine which cities are most vulnerable in a tsunami incident is the object of section 2.1.

After a vulnerable city is determined, a reciprocal GF is calculated by assuming zero free-surface elevation everywhere except for the coast of this city, where the velocity is still zero but the free-surface elevation is unity. The response of all ocean points in the computational domain is the desired GF and the computation is carried out by a shallowwater-wave Cornell Multigrid Coupled Tsunami model (COMCOT; Liu et al. 1998) which will be introduced in section 2.2. Section 2.3 will offer a detailed discussion of how the reciprocal GF is used to evaluate the location of tsunami sensors. The length scale of the discretized GF will be shown to be unimportant in section 2.4.

\subsection{Most Vulnerable Cities According to Hydrodynamic Simulation}

Most of the east coast of Taiwan has a steep sea bottom and the shoaling effect is less significant. An incident tsunami usually does not have time and space to evolve and shoal before it is reflected by the shoreline. To determine 


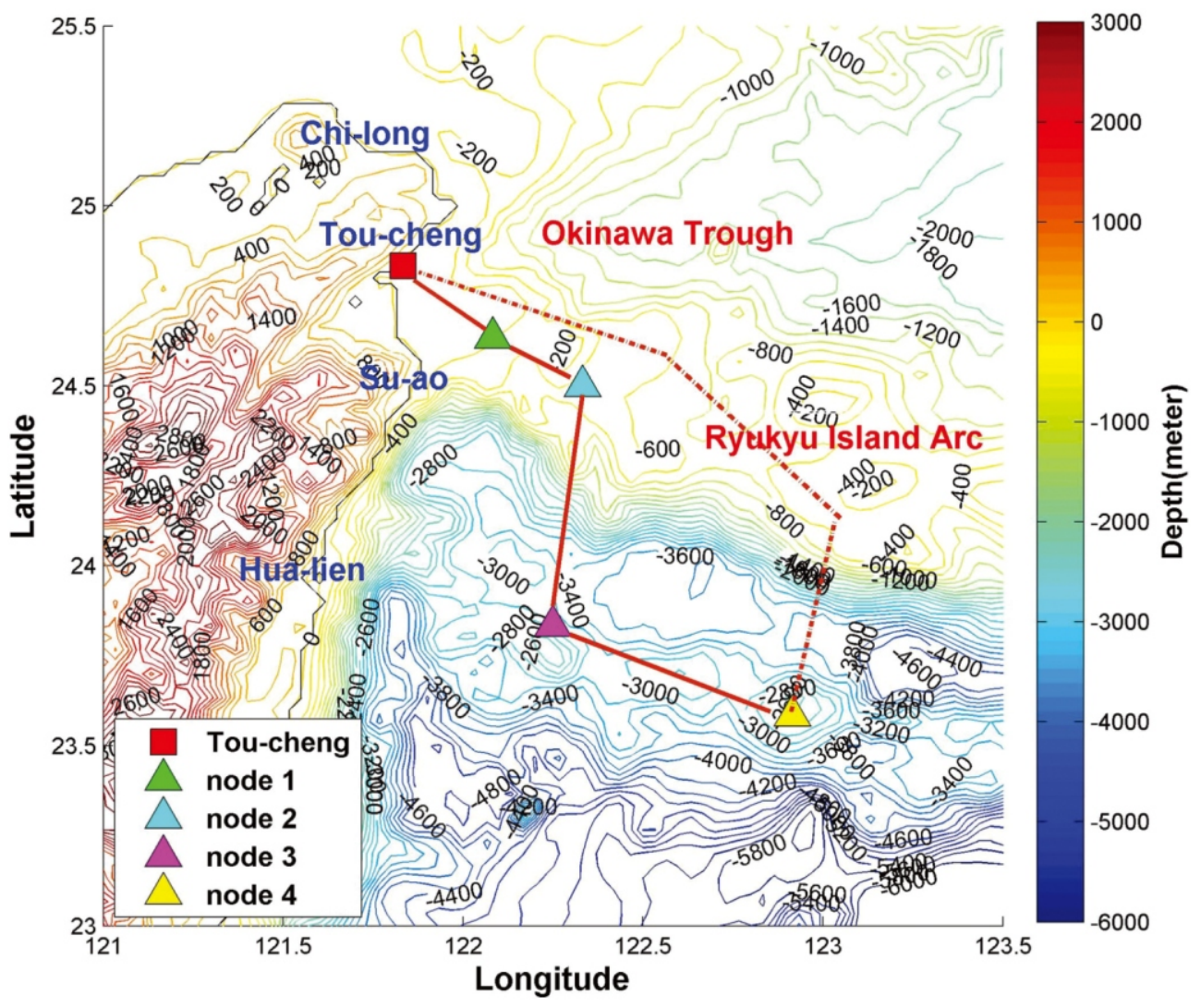

Fig. 1. The preliminary cable route and four nodes of MACHO project. Vulnerable coastal cities in the northeast coast of Taiwan are also shown.

Table 1. The max gain for four nodes with the source located at four vulnerable coastal cities Chi-long, Tou-cheng, Su-ao, and Hua-lien.

\begin{tabular}{lcccc}
\hline Vulnerable city & $\begin{array}{c}\text { node } \mathbf{1} \\
\mathbf{( 1 2 2 . 0 6}^{\circ} \mathbf{E}, \mathbf{2 4 . 6 3 3}\end{array}$ & $\begin{array}{c}\text { node } \mathbf{N}) \\
\left(\mathbf{( 1 2 2 . 3 3 3}^{\circ} \mathbf{E}, \mathbf{~ 2 4 . 5}^{\circ} \mathbf{N}\right)\end{array}$ & $\begin{array}{c}\text { node 3 } \\
\left(\mathbf{( 1 2 2 . 2 3 3}^{\circ} \mathbf{E}, \mathbf{~ 2 3 . 8 3 3}^{\circ} \mathbf{N}\right)\end{array}$ & $\begin{array}{c}\text { node 4 } \\
\left(\mathbf{( 1 2 2 . 9 3 3}^{\circ} \mathbf{E}, \mathbf{~ 2 3 . 5 6 7}^{\circ} \mathbf{N}\right)\end{array}$ \\
\hline Tou-cheng & 0.058 & 0.035 & 0.005 & 0.005 \\
Chi-lung & 0.013 & 0.006 & 0.001 & 0.002 \\
Su-ao & 0.129 & 0.038 & 0.009 & 0.006 \\
Hua-lien & 0.046 & 0.029 & 0.027 & 0.021 \\
\hline
\end{tabular}

which city might be protected by a steep bathymetry and which city might be vulnerable to a devastating tsunami, a numerical hydrodynamic simulation is executed for the entire east coast of Taiwan.

By assuming a solitary wave incident from the Pacific Ocean with a length of the wave crest longer than the length of the Island of Taiwan, the amplification due to shoaling effects can be clearly observed. As is typical for tsunamis propagating in the deep ocean, the incident wave is one meter high which is given via the surface elevation in the east boundary. The resolution of the bathymetry is two minutes, appropriate for the deep Pacific Ocean and the steep sea bottom off the east coast of Taiwan.
As is shown in Fig. 2, the maximum surface elevation is highest in the vicinity of Tou-cheng. Also note that the city of Chi-long, the location of the largest harbor in northern Taiwan, has significant amplification despite its designation as a zone sheltered from the incident wave. Therefore, Toucheng and Chi-long are most vulnerable to a tsunami hazard and should be considered in detail in the deployment of tsunami sensors and seismometers in an observation project.

\subsection{Numerical Hydrodynamic Simulation}

In this study, tsunami propagation is simulated by the COMCOT validated tsunami simulation model (Liu et al. 


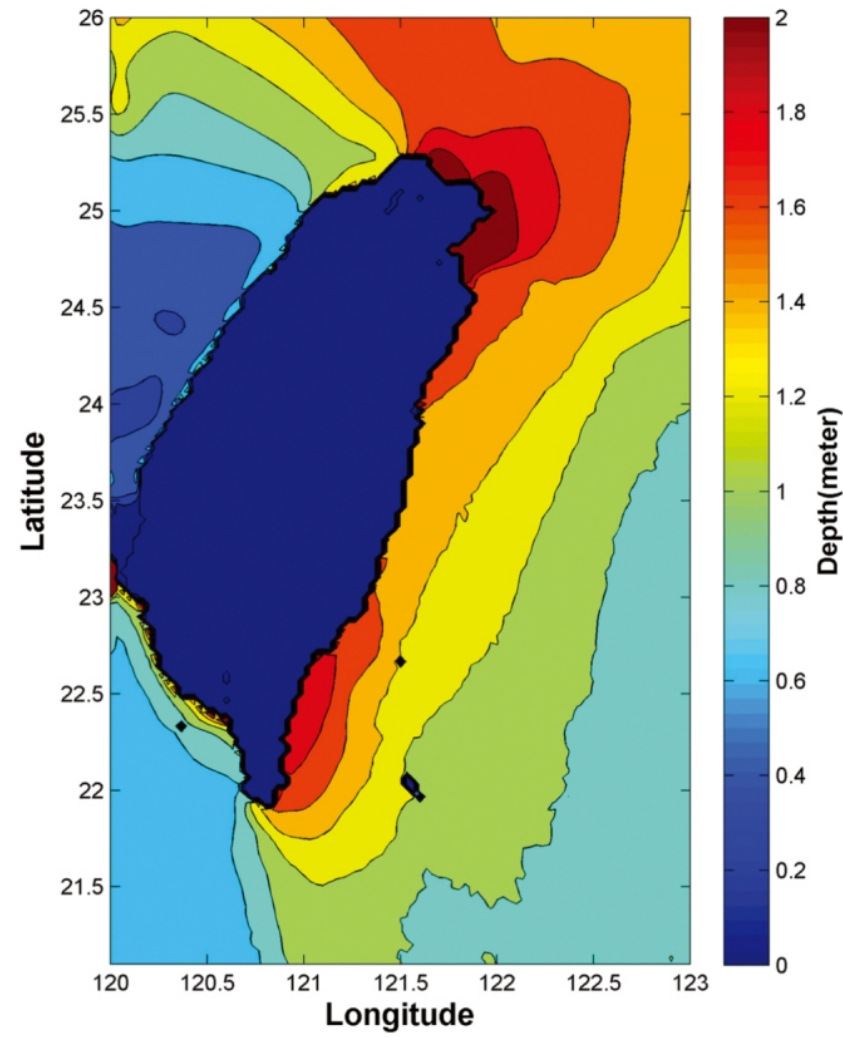

Fig. 2. For a solitary wave of unit amplitude incident from the Pacific Ocean, the max surface elevation is largest in the vicinity of Tou-chen.

1998) to solve both linear and nonlinear shallow water equations. As is explained in section 1.1, a tsunami can be adequately described by linear shallow water equations in ocean water deeper than $50 \mathrm{~m}$ (Shuto 1991). Therefore, nonlinearity and friction are negligible in the offshore region where the GF is computed. Only linear shallow water equations shown below are used in the present study:

$S_{t}+Q_{x}+R_{y}=0$

$Q_{t}+g d s_{x}=0$

$R_{t}+g d s_{y}=0$

where $S$ is the surface elevation, $Q$ and $R$ are fluxes along the $x$ and $y$ directions, and $d$ the water depth. A modified leap-frog finite difference scheme is adopted to solve the equation set. For an arbitrary bathymetry, there is no closed form solution for the Green's function.

It should be noted that the radiation condition (Chapter 5 of Mei 1989) is used in COMCOT to eliminate reflections from the boundary of the computational domain. However, as has been pointed out in Chen et al. (2004), the radiation condition is not applicable to edge waves because the wavenumber is different from that of the plane wave which is used in the radiation condition. To avoid the energy accumulation of an edge wave along the coastline, the computational domain should include the whole island of Taiwan.

\subsection{Reciprocal GF and the Tsunami Vulnerability}

The traditional and reciprocal GF's are symmetric with respect to the source and the receiver (Loomis 1979). The reciprocal GF $G_{A}^{B}$ for tsunami from the hazard-prone city $B$ to point $A$ in the ocean is exactly the response at the city $B$ to a forcing of unit elevation at $A$, or $G_{A}^{B}=G_{B}^{A}$.

This reciprocity of GF can be understood physically as indicating that a linear wave propagating from $B$ to $A$ takes the same time and undergoes the same transformation as it does propagating from $A$ to $B$. As the numerical experiment in Fig. 3 shows, in spite of the inevitable discretization error, the GF from $B$ to $A$ is basically the same as the GF from $A$ to $B$.

The reciprocal GF $G_{A}^{B}$ for each ocean point $A$ is a time series of surface elevation, as is shown in Fig. 5a; both positive and negative GF values are possible. A real tsunami has either a positive or negative elevation in the source zone; hence, both positive and negative GF values are important relative to the tsunami height in the hazard-prone coast. Following Xu (2007), the max absolute value of the reciprocal GF is the max gain for every point in the ocean.

For example, if the max gain of an observatory at point $A$ is small, the observations there are not very useful for the mitigation at the hazard-prone city $B$. On the other hand, if the max gain of an observatory at point $A$ is relatively high, then either the tsunami height or the bottom movement due to earthquake is closely related to the tsunami height at the hazard-prone city $B$. Therefore, according to the max gain derived from the reciprocal GF, the optimum location for an observatory can be determined.

\subsection{Length Scale of the Discretized GF}

The numerical GF computed with an initial unit height at the source has a length scale of a numerical grid; one may ask if the discretized GF exhibits the scale characteristics of the initial elevation and wonder if the applicability of the numerical GF is limited.

To find out the applicability, the discretized GF is used to forecast the offshore tsunami height. The same initial vertical displacement as the direct numerical simulation is multiplied by the corresponding discretized reciprocal GF, and then superimposed over the whole fault zone. As is shown in Fig. 4, the discretized GF gives very accurate tsunami elevation compared with the direct tsunami simulation and reproves yet again that the reciprocity of GF and the discretization of GF does not restrict its applicability for different wavelengths. 


\section{APPLICATION TO THE NORTHEAST COAST OF TAIWAN}

In section 2.1, Tou-cheng and Chi-long are identified as the most vulnerable cities for tsunami hazard on the northeast coast of Taiwan. These two cities should be considered as the key targets to be protected by the MACHO project. The results will be given in sections 3.1 and 3.2 which fol- low. Besides, vulnerability of Su-ao and Hua-lien as well as the commercial harbors nearby can be used as an auxiliary indicator to evaluate the effectiveness of the observatory in section 3.3. The suggestions to the MACHO project based on the analysis are given in section 3.4.

The following analysis is according to the max gain of the reciprocal GF with the hazard-prone coastal city as the source. For the purpose of tsunami early warning, the results are ap-

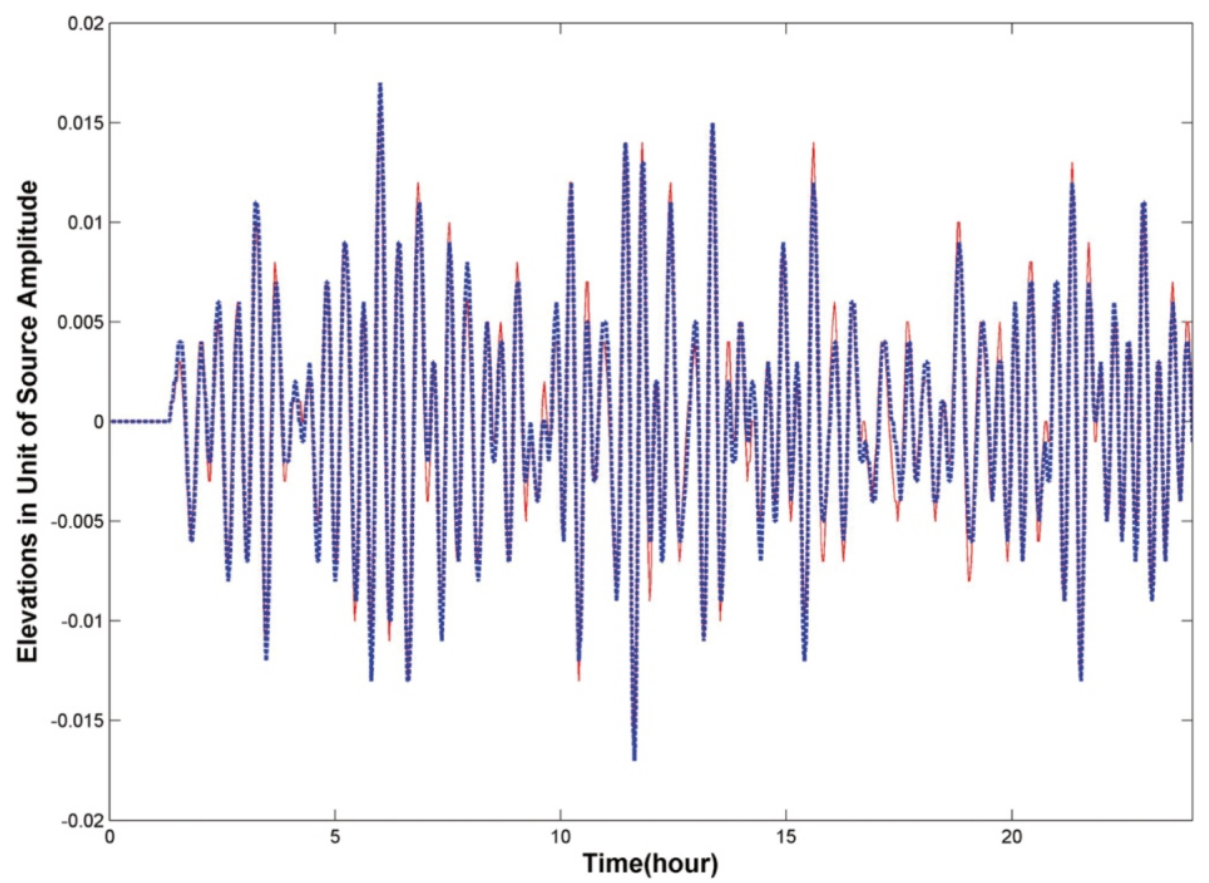

Fig. 3. In spite of the inevitable discretization error, it is evident that the GF from point $B$ to point $A$ (dashed blue line) is the same as the GF from point $A$ to point $B$ (solid red line).

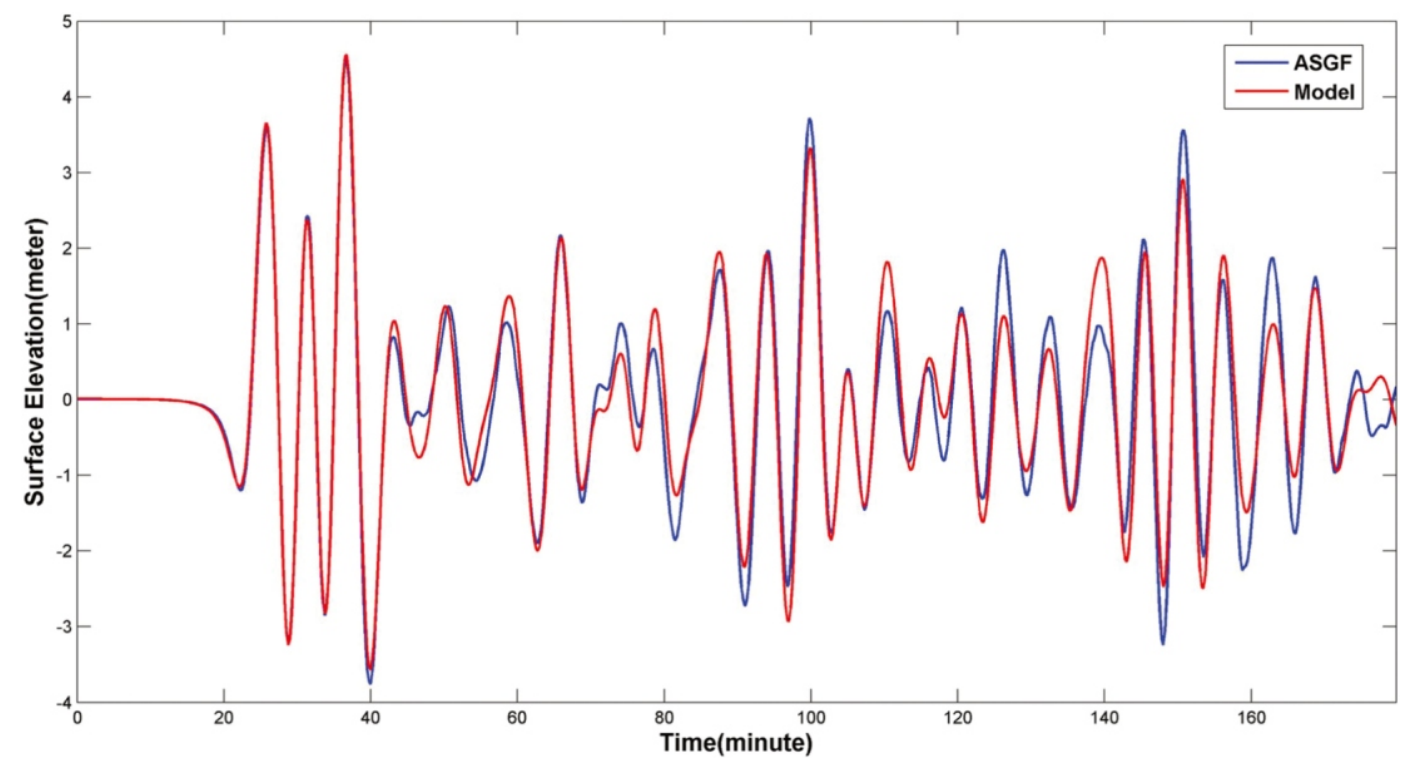

Fig. 4. Tsunami height calculated by multiplying the discretized reciprocal GF and the corresponding vertical displacement superimposed over the whole fault zone (blue line) is very accurate compared with the direct tsunami simulation (red line). 
plicable to both tsunami sensors and seismometers because the later can observe the bottom movement due to earthquake which correlates with the tsunami height in the source region.

\subsection{Tou-Cheng}

The reciprocal GF for the location of four nodes in MACHO project with source located at Tou-cheng is plotted in Fig. 5. As Fig. 5 shows, the max gain is the max absolute variation of surface elevation and its value at node 1 is 0.058 . The max gain for the other three nodes are 0.035 , 0.005 , and 0.005 , respectively. Compared with the max gain diagram of the reciprocal GF shown in Fig. 6a, the max gain at the Yaeyama Ridge region is only one tenth of the max gain at some regions of the Ryukyu Island Arc. From the hydrodynamic point of view, the Ryukyu Island Arc is much more important than the Yaeyama Ridge.

The first two nodes are located at the Ryukyu Island Arc, but the location is not optimized if it is evaluated by the max gain diagram of Fig. 6a. If both the first and second nodes are shifted northwestward, the max gain will be even double the original value.

It should be noted that the max gain at some coastal region near Tou-cheng and Hua-lien is larger than that in the Ryukyu Island Arc. However, these nearshore regions cannot provide enough time for early warning. On the other hand, the Ryukyu Island Arc is far from the shoreline and is practical for the early tsunami warnings.

\subsection{Chi-Long}

Chi-long is the largest harbor in northern Taiwan and the population is more than the sum of the population of all the three cities of Tou-cheng, Su-ao and Hua-lien in the northeast. The numerical shoaling experiment of section $2.1 \mathrm{im}-$ plies that, although Chi-long is on the leeward side, it is vulnerable to a tsunami from the east coast of Taiwan. Therefore, the city of Chi-long should be considered as one of the vulnerable cities for tsunami in the northeast of Taiwan.

The reciprocal GF for four nodes of MACHO with source located at Chi-long is calculated and the max gain for four nodes are $0.013,0.006,0.001$, and 0.002 , respectively. Similar to Tou-cheng, the max gain of Chi-long at the Ryukyu Island Arc region is significantly larger than most offshore regions, as is shown in Fig. 6 b.

\subsection{Su-Ao and Hua-Lien}

Su-ao and Hua-lien are protected from an incident tsunami by the steep bottom slope, as has been discussed in section 2.1. Still they are important harbors in eastern Taiwan and play an important role in commercial activities. The max gains of these two cities are used as auxiliary criteria for the evaluation of tsunami sensor locations.

The reciprocal GF for four nodes of MACHO with sources located at the four vulnerable coastal cities of Toucheng, Chi-long, Su-ao, and Hua-lien are used to obtain the max gain for the four nodes given in Table 1. The max gain value at node 1 is significantly larger for the GF at Su-ao, partly because of the shorter distance to the node.

However, the max gain diagrams in Figs. 6c and d suggest that the location of these nodes is not optimized for Su-ao and Hua-lien. Similar to Tou-cheng and Chi-long, the max gain at some regions of the Ryukyu Island Arc is sig-
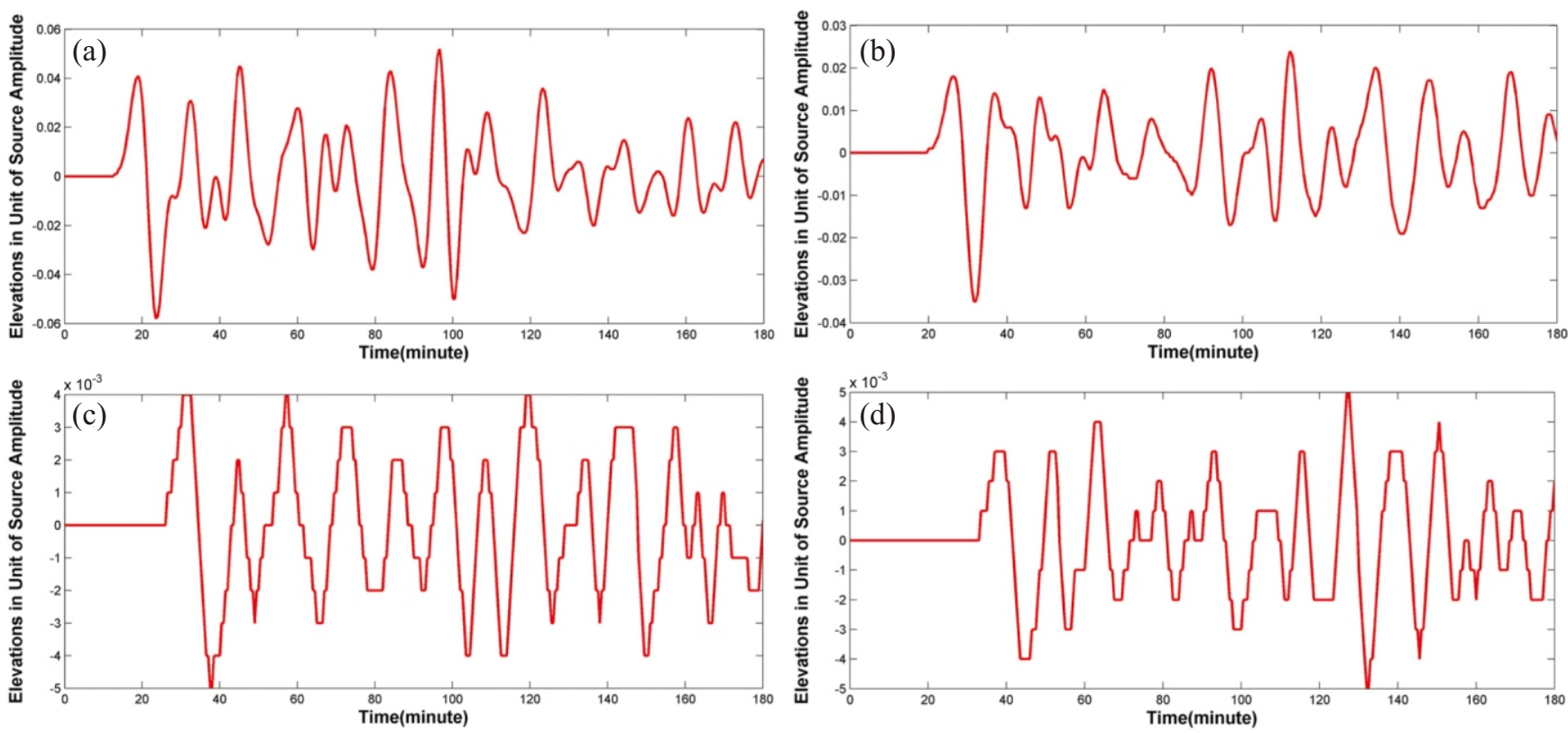

Fig. 5. The time series of the reciprocal GF originates from Tou-cheng to (a) node 1, (b) node 2, (c) node 3, and (d) node 4. 
(a)

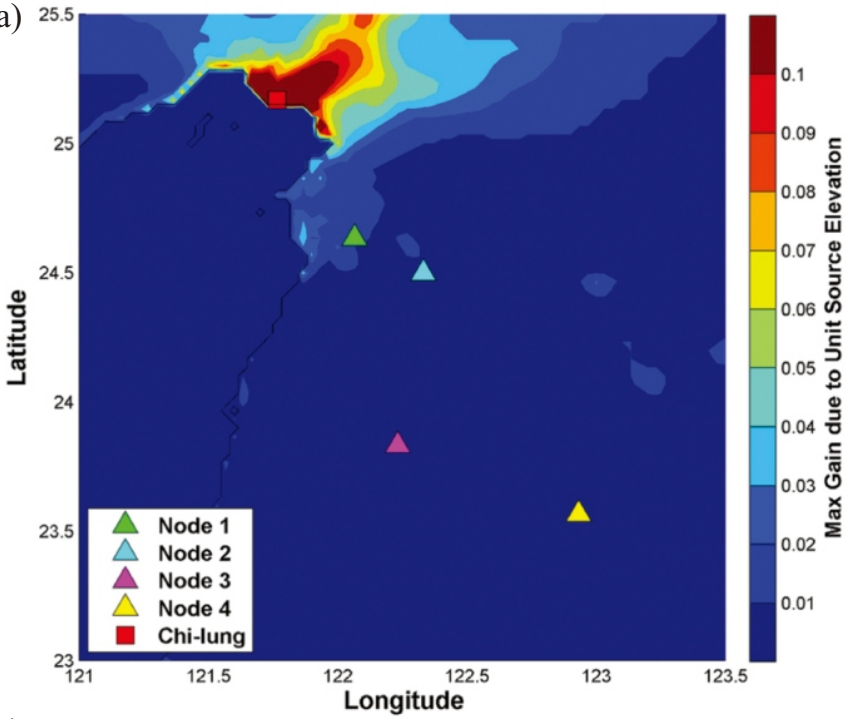

(c)

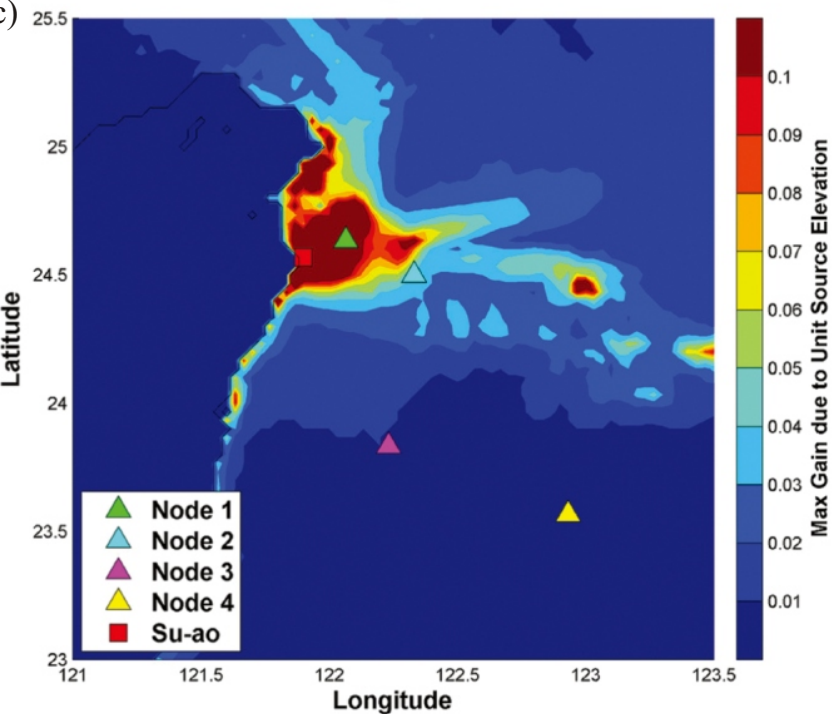

(b)

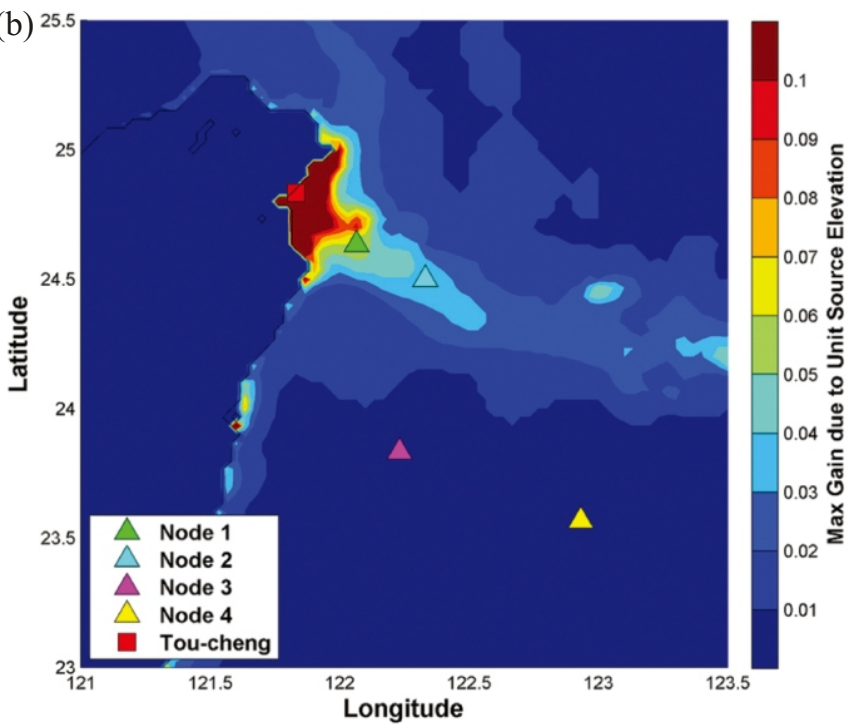

(d)

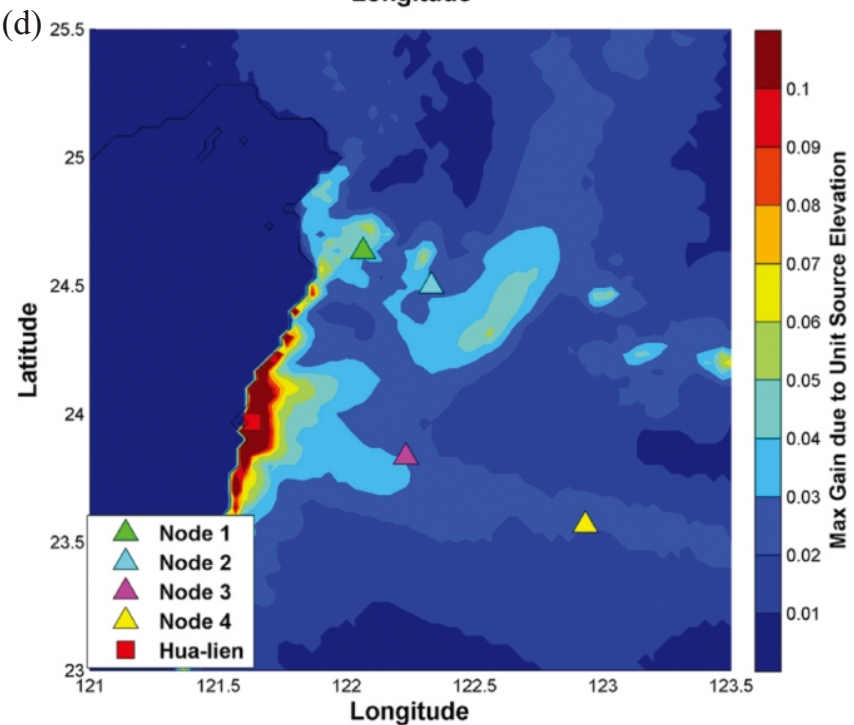

Fig. 6. The max gain of the reciprocal GF originates from (a) Tou-cheng, (b) Chi-long, (c) Su-ao, and (d) Hua-lien.

nificantly larger than most offshore regions.

The Ryukyu Island Arc has much higher gain value than the Yaeyama Ridge, which can be understood in the following way: The Ryukyu Island Arc is shallower than the Yaeyama Ridge; as is shown in Fig. 8, incoming tsunami wave rays from the shallower region of the Ryukyu Island Arc tend to refract toward the vulnerable coast and hence the max gain at the point behind the seamount is amplified. Therefore, a tsunami generated in the Ryukyu Island Arc is more devastating than tsunamis generated in the Yaeyama Ridge. Thus makes the Ryukyu Island Arc better than the Yaeyama Ridge for the deployment of tsunami sensors.

\subsection{Suggestions on the Node Locations and the Cable Route}

From the max gain diagrams shown above, the Ryukyu
Island Arc is more efficient than the Yaeyama Ridge from a hydrodynamic point of view. It is thus suggested that the third and fourth nodes at the Yaeyama Ridge be moved to the Ryukyu Island Arc, unless there are seismological indications that Yaeyama Ridge has a high potential for large earthquakes. If so, the efficiency can still be improved by slightly changing the locations without changing the cable route: According to Figs. 6a - d, if the third node is shifted eastward and the fourth node is shifted westward, the max gain will be increased significantly.

Although the overall max gain in the Ryukyu Island Arc region is relatively large, the value varies from place to place. For example, the first two nodes are on the Ryukyu Island Arc, but the locations are not optimal for the protection of Tou-cheng. To optimize the location, the max gain for the vulnerable cities discussed above is lumped together. It is found that the value for Chi-long is smaller than the other 
three GF's, but the place where the max gain in the Ryukyu Island Arc region is large is very similar. We hence draw only the lowest value for the max gain of the other three GF's in Fig. 7 to determine the location that has largest max gain value for all vulnerable cities.

As has been discussed in section 3.1, max gain for Toucheng will be two times larger if both the first and second nodes are shifted northwestward. For the other three vulnerable cities, the max gain also increases. As can be inferred from Fig. 7, points $a$ and $b$ should be better locations for the first two nodes. If so, the cable route has to be changed slightly, but the increase in the max gain is large.

If modification of the cable route is feasible, further extending the cable eastward to point $d$ in the Ryukyu Island Arc is suggested: The node farther away from the coast of Taiwan will increase the time for an earlier warning; furthermore, the dislocation and water surface elevation at $d$ has a stronger relationship to the tsunami height at the vulnerable cities along the northeast coast of Taiwan. If extending the cable route eastward is not possible, an alternative is to move a node to point $c$ in Fig. 7 to acquire a lengthier early-warning time and higher max gain value. The max gains for points a - $\mathrm{d}$ suggested by the present methodology with the longitude and latitude are shown in Table 2.

\section{SUMMARY AND CONCLUSIONS}

In the present study, the location of tsunami sensors is evaluated according to the max gain of the reciprocal GF based on vulnerable cities. If the max gain of an observatory at point $A$ is relatively high, then either the tsunami height or the bottom movement due to earthquake has high correlation with the tsunami height at the hazardprone coastal city. Therefore, tsunami sensors and seismometers deployed at $A$ are efficient from the hydrodynamic point of view.

In principle, the methodology can be applied to all tsunami observation projects to optimize the usefulness of the tsunami monitoring system. To show the applicability of the methodology, the MACHO project along the Ryukyu Island

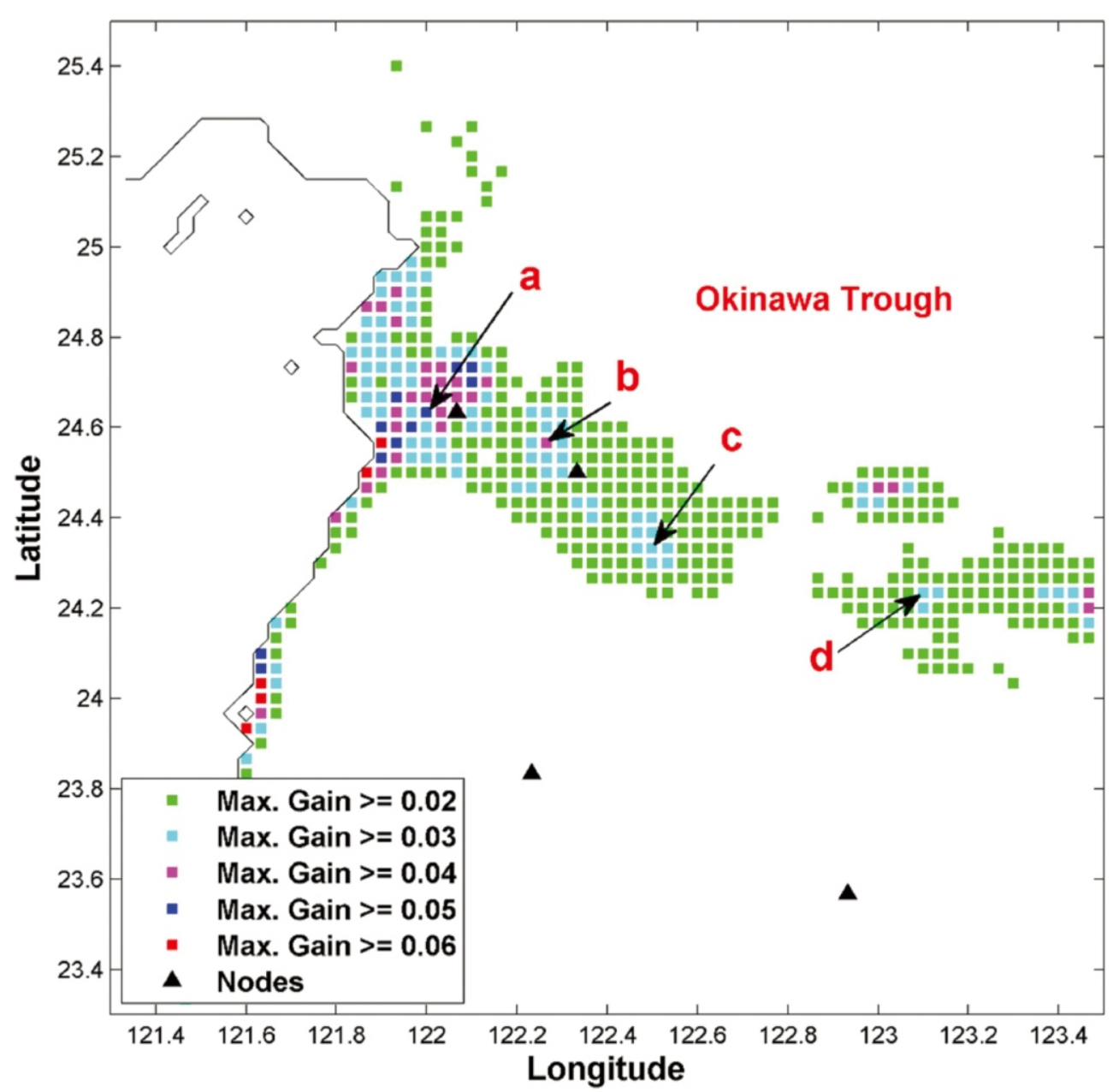

Fig. 7. The lowest value for the max gain of the reciprocal GF of Tou-cheng, Su-ao, and Hua-lien. The value for Chi-long is smaller, but the place where the max gain is large is similar in the Ryukyu Island Arc region. Points $a$ - $d$ have higher gains for each of the vulnerable cities and should be considered as new node locations. 
Table 2. The max gain for four points $a-d$ suggested by the present methodology with source located at four vulnerable coastal cities.

\begin{tabular}{lcccc}
\hline \multirow{2}{*}{ vulnerable city } & $\begin{array}{c}\boldsymbol{a} \\
\left.\mathbf{( 1 2 2 . 0 6 7}^{\circ} \mathbf{E}, \mathbf{~ 2 4 . 6 3 3}^{\circ} \mathbf{N}\right)\end{array}$ & $\begin{array}{c}\boldsymbol{b} \\
\left(\mathbf{1 2 2 . 3 3 3}^{\circ} \mathbf{E}, \mathbf{2 4 . 5}^{\circ} \mathbf{N}\right)\end{array}$ & $\begin{array}{c}c \\
\left(\mathbf{1 2 2 . 2 3 3}^{\circ} \mathbf{E}, \mathbf{~ 2 3 . 8 3 3}^{\circ} \mathbf{N}\right)\end{array}$ & $\begin{array}{c}\boldsymbol{d} \\
\left(\mathbf{1 2 2 . 9 3 3}^{\circ} \mathbf{E}, \mathbf{~ 2 3 . 5 6 7}^{\circ} \mathbf{N}\right)\end{array}$ \\
\hline Tou-cheng & 0.06 & 0.047 & 0.031 & 0.03 \\
Chi-lung & 0.017 & 0.01 & 0.006 & 0.01 \\
Su-ao & 0.17 & 0.097 & 0.037 & 0.041 \\
Hua-lien & 0.052 & 0.042 & 0.044 & 0.045 \\
\hline
\end{tabular}

\section{Vulnerable Coast}

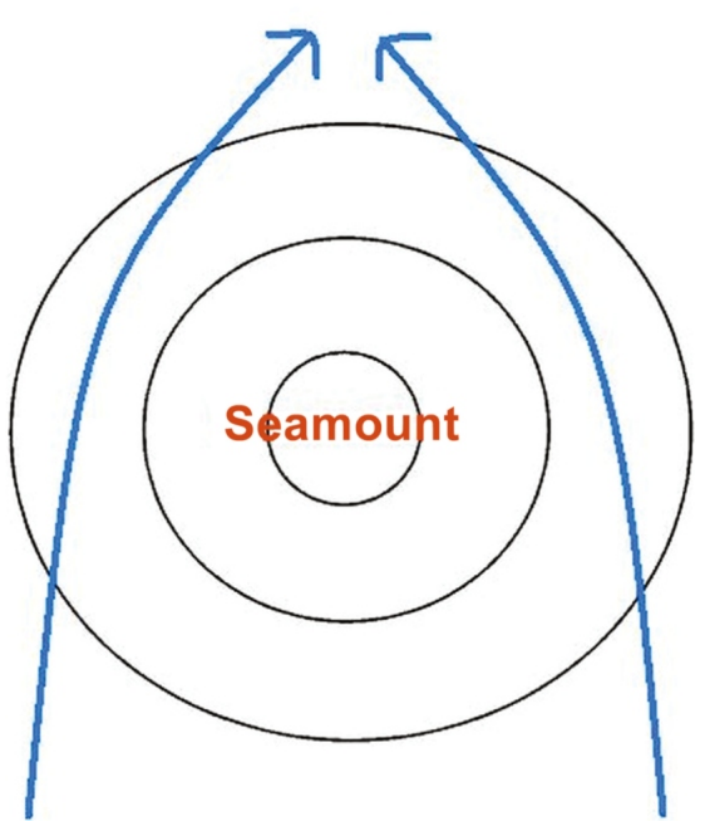

Fig. 8. Incoming wave rays from the shallower region of the Ryukyu Island Arc tend to refract toward the vulnerable coast and hence the max gain at the point behind the seamount is amplified.

Arc and the Yaeyama Ridge in the northeast of Taiwan is analyzed in detail.

In the example in northeastern Taiwan, the four vulnerable cities of Tou-cheng, Chi-long, Su-ao, and Hua-lien are chosen. It is found that for each of the four vulnerable cities, the Ryukyu Island Arc has much higher gain value than the Yaeyama Ridge. Several suggestions based on the max gain distribution are made on the modification of the cable route and node locations where the tsunami sensors and seismometers can be deployed and connected to the cable. Some modifications on the cable route may change the deployment and operating cost, while some modifications on the nodal location do not change the route. In the later case, the cost does not significantly change but the efficiency of the project will be improved.

Acknowledgements This research was completed with grants from Aim for the Top University Plan from the Ministry of Education (96C030227), the National Science Council (NSC97-2611-M-110-014), and the water Resources Agency (MOEAWRA0970305) of Taiwan, Republic of China.

\section{REFERENCES}

Chen, G. Y., C. C. Chien, C. H. Su, and H. M. Tseng, 2004: Resonance induced by edge waves in Hua-Lien Harbor. J. Oceanogr., 60, 1035-1043, doi: 10.1007/s10872-0050011-9. [Link]

Hsu, S. K., C. S. Lee, T. C. Shin, C. S. Liu, B. S. Huang, B. Y. Kuo, C. H. Lin, D. Tang, H. Y. Chang, and C. H. J. Kuo, 2007: Marine Cable Hosted Observatory (MACHO) Project in Taiwan, UT07+SSC07, Tokyo, Japan, 17-20 April 2007. [Link]

Liu, P. L.-F., S. B. Woo, and Y. S. Cho, 1998: Computer programs for tsunami propagation and inundation. School of Civil Environmental Engineering, Cornell University, Ithaca, NY 14853, USA.

Loomis, H. G., 1979: Tsunami prediction using the reciprocal property of Green's functions. Mar. Geodesy, 2, 27-39, doi: 10.1080/15210607909379333. [Link]

Mei, C. C., 1989: The Applied Dynamics of Ocean Surface Waves, Advanced Series on Ocean Engineering, Volume 1, World Scientific, Singapore.

Satake, K., 1987: Inversion of tsunami waveforms for the estimation of a fault heterogeneity: Method and numerical experiments. J. Phys. Earth, 35, 241-254.

Shuto, N., 1991: Numerical simulation of tsunamis - Its present and near future. Nat. Hazards, 4, 171-191, doi: 10.1007/ BF00162786. [Link]

Xu, Z., 2007: The all-source Green's function and its applications to tsunami. Sci. Tsunami Hazards, 26, 59-69. 